\title{
Entropic Upper Bound on Gravitational Binding Energy
}

\author{
C. Vignat ${ }^{1}$, A. Plastino ${ }^{2}$ and A.R. Plastino ${ }^{2,3}$ \\ ${ }^{1}$ L.S.S., Supelec, Universit d'Orsay, Paris, France, EU \\ ${ }^{2}$ National University La Plata, CREG-UNLP-CONICET, \\ Casilla de Correos 727, 1900 La Plata, Argentina \\ ${ }^{3}$ Instituto Carlos I de Física Teórica y Computacional and \\ Departamento de Física Atómica, Molecular y Nuclear, \\ Universidad de Granada, Granada, Spain, EU
}

\begin{abstract}
We prove that the gravitational binding energy $\Omega$ of a self gravitating system described by a mass density distribution $\rho(\mathbf{x})$ admits an upper bound $B[\rho(\mathbf{x})]$ given by a simple function of an appropriate, non-additive Tsallis' power-law entropic functional $S_{q}$ evaluated on the density $\rho$. The density distributions that saturate the entropic bound have the form of isotropic $q$-Gaussian distributions. These maximizer distributions correspond to the Plummer density profile, well known in astrophysics. A heuristic scaling argument is advanced suggesting that the entropic bound $B[\rho(\mathbf{x})]$ is unique, in the sense that it is unlikely that exhaustive entropic upper bounds not based on the alluded $S_{q}$ entropic measure exit. The present findings provide a new link between the physics of self gravitating systems, on the one hand, and the statistical formalism associated with non-additive, power-law entropic measures, on the other hand.
\end{abstract}




\section{INTRODUCTION}

The application of non-additive, power law entropic functionals (and the concomitant $q$-maxent distributions) to the study of self-gravitating systems has attracted considerable interest in recent years [1-11]. In particular, the above mentioned entropic measures and the associated $q$-maxent distributions were found to be relevant for the study of astrophysical self-gravitating systems such as galaxies and galaxy clusters [12-18] and of other astrophysical problems [19-24], such as the statistical characterization of the distribution of stellar rotational velocities in star clusters, where $q$-maxent distributions were successfully applied by Carvalho, do Nascimento, Silva and De Medeiros [19-21].

Compelling evidence for a connection between the physics of self-gravitating systems, on the one hand, and the statistical formalism associated with Tsallis non-additive power law entropy $S_{q}[25$ 27], on the other one, was provided by the long term, $N$-body numerical simulations performed by Taruya and Sakagami [7]. These authors studied the behaviour of a stellar system confined within an adiabatic wall that evolves from initial conditions not described by the Boltzmann-Gibbs distribution and found that, before entering the gravothermally unstable regime, the evolving self-gravitating system can be described remarkably well by a sequence of polytropic distributions with a time dependent polytropic index. These polytropic distributions have precisely the form of Tsallis' $q$-maxent distributions maximizing an $S_{q}$ entropy under the constraints imposed by the total mass and total energy of the self-gravitating system, as was first pointed out in [28]. Taruya and Sakagami also reported numerical evidence indicating that the system exhibits the same kind of behavior even if the simulations are performed without an outer boundary.

Exact results concerning the equation of state within Tsallis thermostatistical framework of a two-dimensional gas of gravitationally interacting particles were obtained by Sau Fa and Lenzi [9]. The Jean's instability of a "gravitational plasma" endowed with a q-gaussian velocity distribution was analyzed by Lima, Silva, and Santos [8]. The gravothermal catastrophe of self-gravitating systems characterized by polytropic (that is, $q$-maxent) distributions was investigated in detail by Taruya and Sakagami (see [5, 6] and references therein). Chavanis and Sire [10] showed that the criterion for nonlinear dynamical stability in the case of spherical stelar systems governed by the Vlasov-Poisson equations exhibits some resemblances with a criterion of thermodynamical stability. On such basis, Tsallis' functional $S_{q}$ can be 
regarded as a useful $H$-function that allows for establishing a continuous connection between stellar polytropes, on the one hand, and isothermal stellar systems on the other one. An increasing body of evidence has been reported in recent years indicating that the polytropic, $q$-maxent distributions are useful to describe dark mater halos of galaxies and galaxy clusters [12 20].

Given a mass density distribution $\rho(\mathbf{x})$ it is intuitively clear that its gravitationally binding energy $\Omega$ tends to decrease as the system becomes less localized. Entropic functionals provide natural quantitative measures of the degree of "delocalization" of the density $\rho$. Consequently, one may expect that there is some relation between the binding energy $\Omega$ and some appropriate entropic functional evaluated on the density $\rho$. The aim of the present work is to prove that such a relation does indeed exist.

\section{TSALLIS STATISTICS AND GRAVITATION}

Some of the most important links between the physics of self-gravitating systems and the statistical formalism based upon Tsallis' entropy come from the study of self-gravitating systems described by the Vlasov-Poisson equations [28]. In order to analyze some important features of galaxies and other self-gravitating systems it is useful to regard them as non-

collisional systems constituted by $N$ particles (the stars) interacting gravitationally [29]. Within this approach, each star moves under the effect of the average gravitational potential $\Phi(\mathbf{x})$ due to the remaining stars in the galaxy. The state of the system is then given by a distribution function $F(\mathbf{x}, \mathbf{v}, t)$ in position-velocity space, where $\mathbf{x}$ denotes a star's position vector, $\mathbf{v}$ stands for its velocity vector and $t$ is the time. The quantity $F(\mathbf{x}, \mathbf{v}, t) d \mathbf{x} d \mathbf{v}$ gives the number of stars having position and velocity vectors respectively within the volume elements $d \mathbf{x}$ and $d \mathbf{v}$ in position and velocity spaces.

The dynamical equation governing the evolution of collisionless system is the Vlasov equation

$$
\frac{\partial F}{\partial t}+\mathbf{v} \cdot \nabla_{x} F-\nabla_{x} \Phi \cdot \nabla_{v} F=0
$$

coupled to the Poisson equation

$$
\nabla^{2} \Phi=4 \pi G \rho
$$


with $\rho(\mathbf{x}, t)=m \int F(\mathbf{x}, \mathbf{v}, t) d \mathbf{v}$, or equivalently to,

$$
\Phi(\mathbf{x}, t)=-G m \int \frac{F\left(\mathbf{x}^{\prime}, \mathbf{v}^{\prime}, t\right)}{\left|\mathbf{x}-\mathbf{x}^{\prime}\right|} d \mathbf{x}^{\prime} d \mathbf{v}^{\prime},
$$

which relates the distribution function $F(\mathbf{x}, \mathbf{v}, t)$ with the Newtonian gravitational potential $\Phi$. Here $G$ denotes Newton's gravitational constant and $m$ is the mass of each star (we assume that all stars have the same mass).

The Vlasov equation can also be cast under the guise $(d F / d t)_{\text {orb. }}=0$. This means that the distribution function $F$ adopts a constant value along the orbit of any star moving in the gravitational potential $\Phi$. It is important to stress that the non-collisional treatment based upon the Vlasov-Poisson system yields only an approximate description of the dynamics of the system. This approximation is, however, a very useful one for various stellar systems [29]. A complete description at the Newtonian level of the evolution of a self-gravitating $N$-particle system is given by the complete set of $N$ coupled Newtonian differential equations of motion corresponding to the $N$ particles. This approach to the dynamics of self-gravitating systems is usually investigated by recourse to numerical $N$-body simulations (see, for instance, [7]).

The total mass,

$$
M=m \int F(\mathbf{x}, \mathbf{v}) d \mathbf{x} d \mathbf{v}
$$

the total energy,

$$
E=\frac{m}{2} \int \mathbf{v}^{2} F(\mathbf{x}, \mathbf{v}) d \mathbf{x} d \mathbf{v}-\frac{G m^{2}}{2} \int \frac{F(\mathbf{x}, \mathbf{v}) F\left(\mathbf{x}^{\prime}, \mathbf{v}^{\prime}\right)}{\left|\mathbf{x}-\mathbf{x}^{\prime}\right|} d \mathbf{x} d \mathbf{v} d \mathbf{x}^{\prime} d \mathbf{v}^{\prime}
$$

and the total angular momentum,

$$
\mathbf{L}=m \int \mathbf{x} \times \mathbf{v} F(\mathbf{x}, \mathbf{v}) d \mathbf{x} d \mathbf{v}
$$

are preserved under the time evolution determined by the coupled Vlasov (11) and Poisson (2) equations. The Vlasov-Poisson dynamical system (1+3) also admits a family of conserved quantities given by functionals of the form

$$
K[F]=\int g(F) d \mathbf{x} d \mathbf{v}
$$

In other words, time dependent solutions $F(\mathbf{x}, \mathbf{v}, t)$ of the Vlasov equation (1) satisfy 


$$
\frac{d K}{d t}=0
$$

When one considers different stationary solutions of the Vlaosv-Poisson system as possible candidates for describing galaxies or other astrophysical $N$-body systems, it is natural to ask which is the distribution function $F(\mathbf{x}, \mathbf{v})$ that maximizes Boltzmann entropy under the constraints given by the total mass (44) and total energy (5) of the system. Unfortunately, the formal solution of this variational problem is given by a distribution function (the isothermal sphere) exhibiting the physically unacceptable features of having infinite mass and infinite energy [29]. On the other hand, the optimization of the non additive Tsallis entropic measure $S_{q}$ under the same constraints yields the stellar polytropic sphere distributions which, for a certain range of values of the entropic parameter $q$, are characterized by a finite mass and a finite total energy [28].

The stellar polytropic sphere distributions in position-velocity space are given by

$$
\begin{aligned}
F(\mathbf{x}, \mathbf{v})=F(\tilde{\epsilon}) & =A\left(\Phi_{0}-\tilde{\epsilon}\right)^{n-3 / 2} & & \tilde{\epsilon} \leq \Phi_{0} \\
& = & 0 & \tilde{\epsilon}>\Phi_{0}
\end{aligned}
$$

where

$$
\tilde{\epsilon}=\frac{1}{2} \mathbf{v}^{2}+\Phi(\mathbf{x})
$$

denotes the total energy (per unit mass) of a single, individual star, and $A$, $\Phi_{0}$, and $n$ (usually referred to as the polytropic index) are appropriate constants. After making the identification of parameters determined by

$$
\frac{1}{1-q}=n-\frac{1}{2}
$$

it can by immediately seen that the stellar polytropic sphere distributions have the $q$-MaxEnt form. That is, they arise from the maximization of the Tsallis' functional

$$
S_{q}[F]=\frac{1}{q-1}\left(1-\int F(\mathbf{x}, \mathbf{v})^{q} d \mathbf{x} d \mathbf{v}\right)
$$

under the constraints given by the total mass (4) and total energy (5). Furthermore, the velocity distribution corresponding to a given space location is a $q$-Gaussian. The isothermal 
sphere distribution is recovered in the limit $n \rightarrow \infty$ (corresponding to $q \rightarrow 1$ ). Another important special case is obtained for $n=5$, which yields the Schuster sphere distribution [29].

For $n<5$ (and $q<7 / 9$ ) the polytropic spheres are systems of finite total mass and energy. The cut-off exhibited by the polytropic distribution (9) corresponds to what is known in the field of non extensive thermostatistics as "Tsallis cut-off prescription". The physical origin of this feature of the stellar polytropic distributions is given by the gravitational escape velocity corresponding to each value of the radial coordinate $r$ [29].

\section{AN ENTROPIC UPPER-BOUND ON GRAVITATIONAL BINDING EN- ERGY}

The gravitational binding energy $\Omega$ of a mass distribution characterized by the density $\rho(\mathbf{x})$ is given by

$$
\Omega=G \int \frac{\rho(\mathbf{x}) \rho\left(\mathbf{x}^{\prime}\right)}{\left|\mathbf{x}-\mathbf{x}^{\prime}\right|} d \mathbf{x} d \mathbf{x}^{\prime}
$$

The system's total gravitational potential energy is equal to $-\Omega$. In the case of a selfgravitating system constituted by $N$ particles of mass $m$ and described by a space-velocity distribution $F(\mathbf{x}, \mathbf{v})$ we have $\rho(\mathbf{x})=\int F(\mathbf{x}, \mathbf{v}) d \mathbf{v}$ and

$$
\Omega=\frac{G m^{2}}{2} \int \frac{F(\mathbf{x}, \mathbf{v}) F\left(\mathbf{x}^{\prime}, \mathbf{v}^{\prime}\right)}{\left|\mathbf{x}-\mathbf{x}^{\prime}\right|} d \mathbf{x} d \mathbf{v} d \mathbf{x}^{\prime} d \mathbf{v}^{\prime} .
$$

It is intuitively clear that the gravitational binding energy decreases as the system's mass distribution $\rho(\mathbf{x})$ becomes more delocalized. Quantitative measures for this degree of delocalization are provided by entropic measures evaluated on a probability density defined as

$$
f(\mathbf{x})=\frac{\rho(\mathbf{x})}{M},
$$

where $M=\int \rho(\mathbf{x}) d \mathbf{x}$ is the total mass of the system. Note that $f(\mathbf{x})$ is normalized to 1. These considerations suggest that there may be a connection between the gravitational binding energy $\Omega$, on the one hand, and an appropriate entropic measure evaluated on the probability density $p(\mathbf{x})$. Here we are going to prove that, indeed, such a connection exists 
and can be established as an entropic upper bound on the binding energy $\Omega$. We are going to obtain an upper bound to $\Omega$ given as a monotonic decreasing function of an appropriate $S_{q}$ entropy evaluated on $f(\mathbf{x})$.

We derive the above mentioned entropic upper bound on $\Omega$ from a special case of the Hardy-Littlewood-Sobolev inequality [30] which reads as follows

Theorem 1. If $q_{1}=\frac{2 D}{2 D-\lambda}$ with $0<\lambda<D$ and $f \in L^{q_{1}}\left(\mathbb{R}^{D}\right)$ then

$$
\left|\int_{\mathbb{R}^{D}} \int_{\mathbb{R}^{D}} f(\mathbf{x}) f(\mathbf{y})\right| \mathbf{x}-\left.\mathbf{y}\right|^{-\lambda} d \mathbf{x} d \mathbf{y} \mid \leq C(D, \lambda)\|f\|_{q_{1}}^{2}
$$

with the constant

$$
C(D, \lambda)=\pi^{\frac{p}{2}} \frac{\Gamma\left(\frac{D-\lambda}{2}\right)}{\Gamma\left(D-\frac{\lambda}{2}\right)}\left(\frac{\Gamma\left(\frac{D}{2}\right)}{\Gamma(D)}\right)^{-1+\frac{\lambda}{D}}
$$

The case of equality is reached if and only if

$$
f(\mathbf{x})=A\left(\gamma^{2}+|\mathbf{x}-\mathbf{a}|^{2}\right)^{-\frac{2 D-\lambda}{2}}
$$

for some $A \in \mathbb{C}, \gamma \in \mathbb{R} \backslash\{0\}$ and $a \in \mathbb{R}^{D}$.

We first remark that this inequality is invariant if $\mathbf{x}$ and $\mathbf{y}$ are shifted by $\mathbf{a}$ so that $\mathbf{a}$ can be assumed equal to 0 without loss of generality. Function $f(\mathbf{x})$ can then be written as

$$
f(\mathbf{x})=A \gamma^{2}\left(1+\frac{|\mathbf{x}|^{2}}{\gamma^{2}}\right)^{-\frac{2 D-\lambda}{2}} .
$$

Since moreover inequality (16) is homogeneous in $f$, we may also assume that

$$
\int_{\mathbb{R}^{D}} f(\mathbf{x}) d \mathbf{x}=1
$$

The probability density (18) is clearly a $q$-Gaussian distribution. We remark moreover that since $0<\lambda<D$,

$$
\frac{D}{2}<\frac{2 D-\lambda}{2}=\frac{1}{1-q_{2}}<D
$$

so that

$$
1-\frac{2}{D}<q_{2}=\frac{2 D-\lambda-2}{2 D-\lambda}<1-\frac{1}{D}
$$


The term $\|f\|_{q_{1}}^{2}$ in the upper-bound of (16) can be recast as,

$$
C\|f\|_{q_{1}}^{2}=C\left(\int_{\mathbb{R}^{D}} f^{q_{1}}(\mathbf{x}) d \mathbf{x}\right)^{\frac{2}{q_{1}}}=C\left(1+\left(1-q_{1}\right) S_{q_{1}}[f]\right)^{\frac{2}{q_{1}}},
$$

which is a monotonically decreasing function of the entropic functional $S_{q_{1}}$ evaluated on the density $f$.

The integral appearing in the left hand side of equation (16) can be interpreted as proportional to the binding energy $\Omega$ of a mass density distribution $\rho(\mathbf{x})=M f(\mathbf{x})$ (with total mass $M$ ) where the interaction of mass elements $\rho\left(\mathbf{x}_{\mathbf{1}}\right) d \mathbf{x}_{\mathbf{1}}$ and $\rho\left(\mathbf{x}_{\mathbf{2}}\right) d \mathbf{x}_{\mathbf{2}}$ separated by a distance $r=\left|\mathbf{x}_{\mathbf{2}}-\mathbf{x}_{\mathbf{1}}\right|$ is described by a potential function behaving as

$$
-\frac{\alpha}{r^{\lambda}} \rho\left(\mathbf{x}_{1}\right) \rho\left(\mathbf{x}_{2}\right) d \mathbf{x}_{1} d \mathbf{x}_{2}
$$

where $\alpha$ is a constant characterizing the "strength" of the above mentioned interaction. The alluded binding energy is then

$$
\Omega=\alpha M^{2} \int_{\mathbb{R}^{D}} \int_{\mathbb{R}^{D}} f(\mathbf{x}) f(\mathbf{y})|\mathbf{x}-\mathbf{y}|^{-\lambda} d \mathbf{x} d \mathbf{y} .
$$

Now, combining equations (16), (23) and (25), we get the desired bound on the binding energy,

$$
\Omega \leq C \alpha M^{2}\left(1+\left(1-q_{1}\right) S_{q_{1}}\left[\frac{\rho(\mathbf{x})}{M}\right]\right)^{\frac{2}{q_{1}}} .
$$

In the case of the gravitational interaction in $D$-dimensional space we have $\lambda=D-2$. Consequently, the entropic parameter $q_{1}$ corresponding to the entropic bound (26) is

$$
q_{1}=\frac{2 D}{D+2}
$$

In order to get some further physical insight on the particular structure of the entropic bound $B$, it is instructive to consider it from the point of view of its scaling behaviour. It is clear that the binding energy $\Omega$ obeys some simple scaling laws. It seems reasonable that a physically meaningful entropic upper bound for $\Omega$ should satisfy the same scaling laws as $\Omega$. This requirement is particularly sensible if the bound is exhaustive, in the sense that there are density distributions that do achieve the bound. Now, a natural candidate for an entropy-related functional of $\rho(\mathbf{x})$ satisfying simple scaling laws is provided by a power-law functional proportional to 


$$
I=\left[\int \rho(\mathbf{x})^{q} d \mathbf{x}\right]^{\kappa} .
$$

The question is now to find the appropriate values of the exponents $q$ and $\kappa$. Let's consider first the scaling transformation $\rho(\mathbf{x}) \rightarrow \tilde{\rho}(\mathbf{x})=l \rho(\mathbf{x})$, with $l>0$ a scaling parameter (note that the mass density $\rho(\mathbf{x})$ is not in general normalized to 1 ). The quantities $\Omega$ and $I$ transform respectively as $\Omega \rightarrow l^{2} \Omega$ and $I \rightarrow l^{\kappa q} I$, which suggests

$$
\kappa q=2 .
$$

On the other hand, consider the transformation $\rho(\mathbf{x}) \rightarrow \tilde{\rho}(\mathbf{x})=\rho(l \mathbf{x})$. In this case $\Omega$ and $I$ behave as $\Omega \rightarrow l^{-2-D} \Omega$ and $I \rightarrow l^{-\kappa D} I$, suggesting,

$$
D+2=\kappa D
$$

Combining (29) and (30) we finally obtain

$$
q=\frac{2 D}{D+2}, \text { and } \kappa=\frac{2}{q}
$$

which are precisely the values of the exponents appearing in the bound (26) $)$. This argument, of course, does not constitute a formal proof of the bound (the proof, as we already explained, is based on the Hardy-Littlewood-Sobolev inequality). However, the above scaling argument suggests that it is unlikely that an entropic bound for the gravitational binding energy can be found which is not based on the functional (28) with the exponents (31). In other word, the entropic bound (26) seems to be unique.

\section{IV. $q$-GAUSSIAN MAXIMIZERS}

Let us consider now the particular case of highest relevance for the study of self gravitating systems, which is the one corresponding to three dimensional space $(D=3)$ and $\lambda=1$, in which case the integral appearing in equation (25) ) has the standard form of the gravitating binding energy of a mass distribution in three dimensional space. In this case we have $q_{1}=6 / 5$ and the entropic upper bound for the gravitational binding energy of a system of total mass $M$ described by a density distribution $\rho(\mathbf{x})$ is, 


$$
\Omega \leq B[\rho(\mathbf{x})]=C(3,1) G M^{2}\left(1-\frac{1}{5} S_{6 / 5}\left[\frac{\rho(\mathbf{x})}{M}\right]\right)^{\frac{5}{3}} .
$$

Let us now consider the density distributions achieving the entropic bound (32). Combining equations (15),(19), and (20) we can write the maximizer mass distributions as

$$
\rho(r)=\frac{3 M}{4 \pi \gamma^{3}}\left(1+\frac{r^{2}}{\gamma^{2}}\right)^{-\frac{5}{2}},
$$

where $r=|\mathbf{x}|$. Now, equation (33) describes the celebrated Plummer density profile, which is well known in astrophysics [29, 31 33]. The parameter $\gamma$ appearing in (33) is the "Plummer radius". It is a scale parameter determining the size of the inner "core" of the mass distribution.

Plummer's density profile is of considerable theoretical interest. It corresponds to a polytropic sphere distribution in position-velocity space with polytropic index $n=5$. This distribution has the remarkable property of constituting the limit case of a polytropic (and a maximum- $S_{q}$ ) distribution exhibiting a finite total mass and therefore being physically plausible. As already mentioned, polytropic spheres with $n \leq 5$ have finite total mass, while those with $n>5$ have infinite mass. Another notable feature of the $n=5$ polytropic sphere is that it is a maximum- $S_{q}$ distribution in position-velocity space that leads to a density distribution (Plummer's distribution) which is itself a $q$-Gaussian (that is, a maximum- $S_{q}$ distribution).

\section{CONCLUSIONS}

In the present work we obtained an entropic upper bound $B[\rho]$ on the gravitational binding energy $\Omega$ associated with a density mass distribution $\rho(\mathbf{x})$. This upper bound is a simple function of an appropriate power-law non-additive Tsallis' entropy $S_{q}$ evaluated on $\rho$. In the case of the standard, Newtonain gravitational interaction in three dimensional space the alluded entropy is characterized by the entropic parameter $q=\frac{6}{5}$.

The density distributions achieving the entropic bound (that is, satisfying $\Omega=B$ ) exhibit

the form of an isotropic $q$-Gaussian distribution with $q=\frac{8}{5}$. These maximizer distributions coincide with the Plummer density profile, well known in astrophysics.

It is well known that many of the important facts concerning gravitation are related to 
simple scaling laws, Kepler's celebrated third law of planetary motion being historically the first example [34]. Here we have provided a heuristic scaling argument suggesting that the entropic bound $B[\rho]$ for the gravitational binding energy is unique, with the specific value of the Tsallis parameter $q_{1}$ appearing in the bound determined by the space dimension $D$.

The present findings constitute intriguing new evidence of the relationship that exists between the physics of self gravitating systems, on the one hand, and the statistical formalism associated with non-additive, power law entropic functionals, on the other hand.

Acknowledgments. This work was partially supported by by the Project FQM-2445 of the Junta de Andalucía and by the Grant FIS2008-2380 of the Ministerio de Innovación y Ciencia, Spain.

[1] N. Komatsu, T. Kiwata and S. Kimura, Phys. Rev. E 82 (2010) 021118.

[2] J.L. Du, Astrophys. and Space Sci. 312 (2007) 47.

[3] J.L. Du, Europhys. Lett. 67 (2004) 893.

[4] R. Silva and J.S. Alcaniz, Physica A 341 (2004) 208.

[5] A. Taruya and M. Sakagami Physica A 307 (2002) 185.

[6] A. Taruya and M. Sakagami, Cont. Mech. and Therm. 16 (2004) 279.

[7] A. Taruya and M. Sakagami, Phys. Rev. Lett. 90 (2003) 181101.

[8] J.A.S. Lima, R. Silva, and J. Santos, Astron. and Astrophys. 396 (2002) 309.

[9] K. Sau Fa and E.K. Lenzi, J. of Math. Phys. 42 (2001) 1148.

[10] P.H. Chavanis and C. Sire, Physica A 356 (2005) 419.

[11] T. Yamano, Phys. Lett. A 264 (1999) 276.

[12] C.J. Saxton and I. Ferreras, Mon. Not. R. Astron. Soc. 405 (2010) 77.

[13] C.J. Saxton and K. Wu, Mon. Not. R. Astron. Soc. 391 (2008) 1403.

[14] J. Calvo, E. Florido, O. Sánchez, E. Battaner, J. Soler and B. Ruiz-Granados, Physica A 388 (2009) 2321.

[15] S.H. Hansen and B. Moore, New Astronomy 11 (2006) 333.

[16] T. Kronberger, M.P. Leubner and E. van Kampen, Astron. and Astrophys. 453 (2006) 21.

[17] M.P. Leubner, Astrophys. J. 632 (2005) L1. 
[18] T. Matos, D. Nez, and R.A. Sussman, Class. and Quantum Gravity 21 (2004) 5275.

[19] J.C. Carvalho, J.D. do Nascimento Jr., R. Silva and J.R. De Medeiros, Astrophys. J. 696 (2009) L48.

[20] J.C. Carvalho, R. Silva, J.D. do Nascimento Jr. and J.R. De Medeiros, Europhys. Lett. 84 (2008) 59001.

[21] B.B. Soares, J.C. Carvalho, J.D. do Nascimento Jr. and J.R. De Medeiros, Physica A 364 (2006) 413.

[22] M.P. Leubner, Astrophys. and Space Sci. 282 (2002) 573.

[23] M.A. Moret, V. de Senna, G.F. Zebende and P. Vaveliuk, Physica A 389 (2010) 854.

[24] C. Tsallis, D. Prato, and A.R. Plastino, Astrophys. and Space Sci. 290 (2004) 259.

[25] C. Tsallis, J. Stat. Phys. 52 (1988) 479.

[26] M. Gell-Mann and C. Tsallis, Eds. Nonextensive Entropy: Interdisciplinary Applications, Oxford University Press, Oxford, 2004.

[27] C. Tsallis, Introduction to Nonextensive Statistical Mechanics: Approaching a Complex World, Springer, New York, 2009.

[28] A. R. Plastino and A. Plastino, Phys. Lett. A 174 (1993) 384.

[29] J. Binney and S. Tremaine, Galactic Dynamics (Princeton University Press, Princeton, 1987).

[30] E. Lieb and M. Loss, Analysis, Graduate Studies in Mathematics, Second Edition, AMS

[31] H.C. Plummer, Mon. Not. R. Astron. Soc. 71 (1911) 460.

[32] H. Dejonghe, Mon. Not. R. Astron. Soc. 224 (1987) 13.

[33] S.J. Aarseth, M. Henon and R. Wielen, Astron. and Astrophys. 37 (1974) 183.

[34] M. Schroeder, Fractals, Chaos, Power Laws, W. H. Freeman, 1992. 